defects: Review of registry of complications and recommendatios to minimize future risk. Catheter Cardiovasc Interv. 2004;63:496-502.

4. DiBardino DJ, McElhinney DF, Kaza AK, et al. Analysis of the US Food and Drug Administration manufacturer and user facility device experience database for adverse events involving Amplatzer Septal Occluder devices and comparison with the Society of Thoracic Surgery Congenital Cardiac Surgery database. J Thorac Cardiovasc Surg. 2009;137:1334-41.

5. Crawford GB, Brindis RG, Krucoff MW, et al. Percutaneous atrial septal occluder devices and cardiac erosion: A review of the literature. Catheter Cardiovasc Interv. 2012;80:157-67.

6. Amin Z. Echocardiographic predictors of cardiac erosion after Amplatzer septal occluder placement. Catheter Cardiovasc Interv. 2014;83:84-92.

7. Diab K, Kenny D, Hijazi ZM. Erosions, erosions, and erosions! Device closure of atrial septal defects: how safe is safe? Catheter Cardiovasc Interv. 2012;80:168-74.

8. Podnar T, Martanovic P, Gavora P, et al. Morphological variations of secundum-type atrial septal defects: Feasibility for percutaneous closure using Amplatzer septal occluders. Catheter Cardiovasc Interv. 2001;53:386-91.

9. OB́yrne ML, Glatz AC, Sunderji S, et al. Prevalence of deficient retro-aortic rim and its effects on outcomes in device closure of atrial septal defects. Pediatr Cardiol. 2014;35:1181-90.

10. Roberts WT, Parmar J, Rajathurai T. Very late erosion of Amplatzer septal occluder device presenting as pericardial pain and effusion 8 years after placement. Catheter Cardiovasc Interv. 2013;82:E592-4.

Alejandro E. Contreras ${ }^{a, *}$, Adolfo Ferrero Guadagnoli ${ }^{\mathrm{b}}$ y Alejandro Peirone ${ }^{b}$

a Servicio de Cardiología, Hospital Privado Universitario de Córdoba, Instituto Universitario de Ciencias Biomédicas de Córdoba, Córdoba, Argentina

b Servicio de Hemodinamia, Hospital Privado Universitario de Córdoba, Instituto Universitario de Ciencias Biomédicas de Córdoba, Córdoba, Argentina

* Autor para correspondencia. Naciones Unidas 346, CP X5016KEH, Barrio Parque Vélez Sársfield, Córdoba, Argentina; Teléfono: +543514688220; Fax: +433514688818.

Correo electrónico: aletreras@hotmail.com

(A.E. Contreras).

http://dx.doi.org/10.1016/j.acmx.2016.05.006 $1405-9940$

( 2016 Instituto Nacional de Cardiología Ignacio Chávez. Publicado por Masson Doyma México S.A. Este es un artículo Open Access bajo la CC BY-NC-ND licencia (http://creativecommons.org/ licencias/by-nc-nd/4.0/).

\section{Bloqueo auriculoventricular completo reversible durante el cierre percutáneo de comunicación interauricular}

\section{Transient complete atrioventricular block during percutaneous atrial septal defect closure}

El bloqueo completo del nódulo auriculoventricular (AV) posterior a un cierre percutáneo de comunicación interauricular tipo ostium secundum es una complicación infrecuente, y la mayoría de los casos se resuelven espontáneamente, siendo rara la necesidad de colocación de marcapasos.

\section{Informe de caso}

Presentamos el caso de un paciente femenino de 5 años de edad (14,3 kg de peso), con una comunicación interauricular tipo ostium secundum de $11 \mathrm{~mm}$ de diámetro mayor por ecocardiografía transtorácica bidimensional, bordes adecuados para cierre percutáneo, dilatación de cavidades cardíacas derechas e hipertensión arterial pulmonar leve (presión pulmonar media de $29 \mathrm{mmHg}$ ). El electrocardiograma muestra ritmo sinusal y conducción AV conservada. Se propone cierre percutáneo, el cual es realizado bajo control angiográfico y por ecocardiografía transtorácica. Durante el procedimiento, y previo a la elección del dispositivo oclusor, se realiza medición del defecto con balón elastomérico según técnica de «stop flow», el cual arroja $15 \mathrm{~mm}$ de diámetro, por lo cual se elige dispositivo NitOcclud $^{\circledR}$ ASD-R (PFM Medical, Alemania) de $16 \mathrm{~mm}$. Se coloca dispositivo sin complicaciones. Inmediatamente después de la apertura del disco derecho del dispositivo, y previa a la liberación del mismo, se observa en monitorización electrocardiográfica, bloqueo AV completo (fig. 1A), por lo que se decide recapturar el dispositivo y suspender el procedimiento. Después de esto, queda bajo control electrocardiográfico y se observa recuperación de la conducción AV hasta llegar a recuperar el ritmo sinusal 72 min después (figs. 1B-D). Se concluye el procedimiento y se decide esperar al crecimiento del niño para nuevo intento de cierre percutáneo.

Treinta y siete meses después del primer procedimiento, a los 7 años de edad ( $21 \mathrm{~kg}$ de peso), se intenta nuevamente el cierre percutáneo del defecto, bajo control ecocardiográfico transesofágico tridimensional (defecto de $12 \mathrm{~mm}$ en su diámetro mayor, fig. 2A). No se realiza medición con balón elastomérico y se intenta el cierre con el dispositivo NitOcclud $^{\circledR}$ ASD-R de $14 \mathrm{~mm}$ (fig. 2B). Se logra cerrar el defecto sin complicaciones y sin alteraciones en la conducción AV. Es dada de alta a las $24 \mathrm{~h}$, con ritmo sinusal y conducción AV conservada. Un nuevo control al mes del procedimiento muestra dispositivo implantado sin fuga residual y electrocardiograma con ritmo sinusal normal (fig. 2C).

\section{Discusión}

La presencia de bloqueo auriculoventricular durante o posterior al cierre percutáneo de una comunicación interauricular es poco frecuente, con una incidencia entre $0,23^{1}$ y $4,9 \%^{2}$. Un estudio retrospectivo que incluyo 610 pacientes, sometidos a cierre de foramen oval permeable y defectos del septum interauricular, demostró un incremento significativo de la duración de la onda $\mathrm{P}$ en electrocardiogramas 


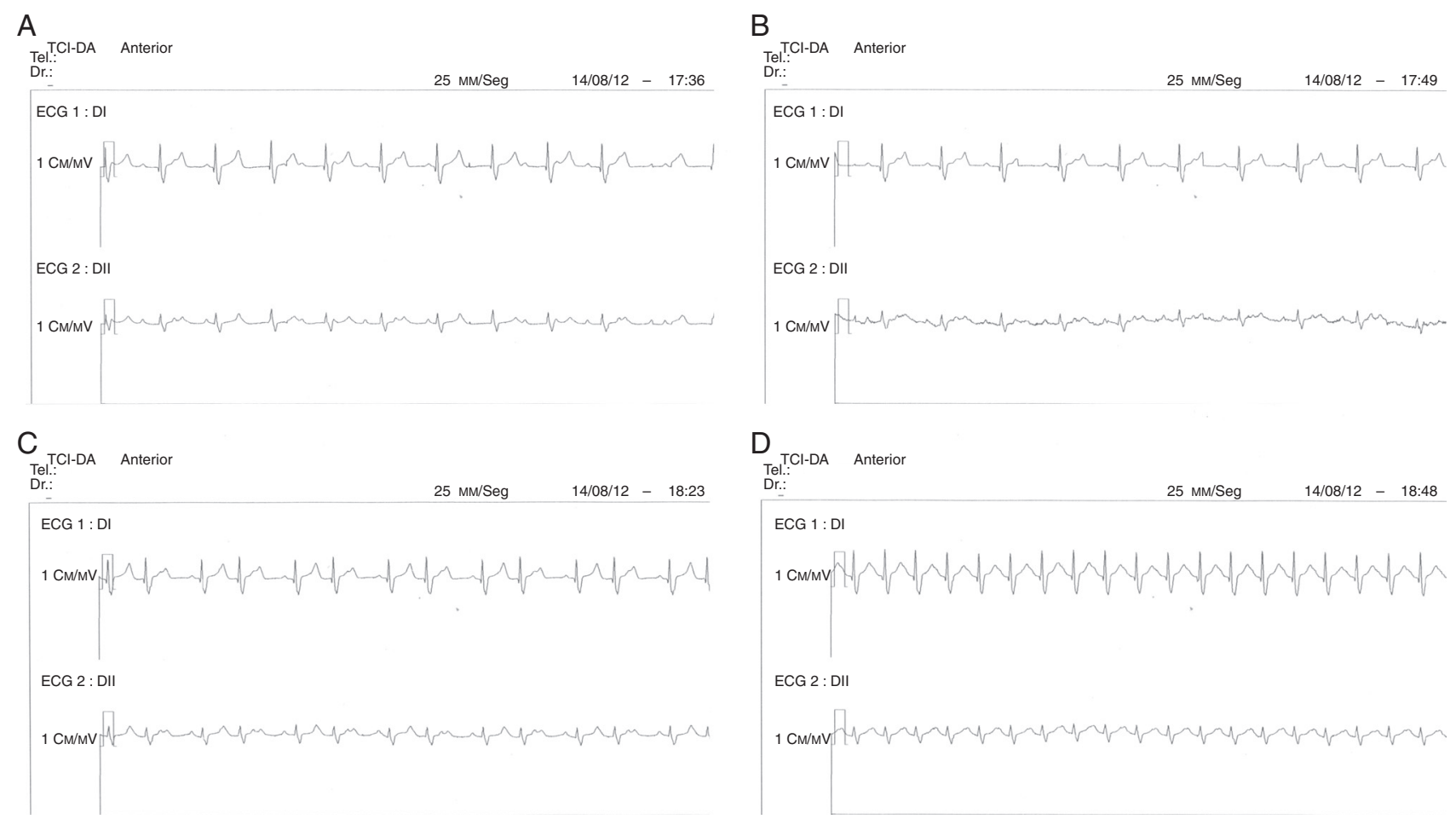

Figura 1 Electrocardiograma en el momento de la apertura del dispositivo: A) Bloqueo AV completo. B) Bloqueo AV de segundo grado, 13 min después de recapturar el dispositivo. C) Bloqueo AV de primer grado tipo, 1, 47 min después de recapturar el dispositivo. D) Taquicardia sinusal con conducción AV preservada, 54 min después de recapturado el dispositivo.
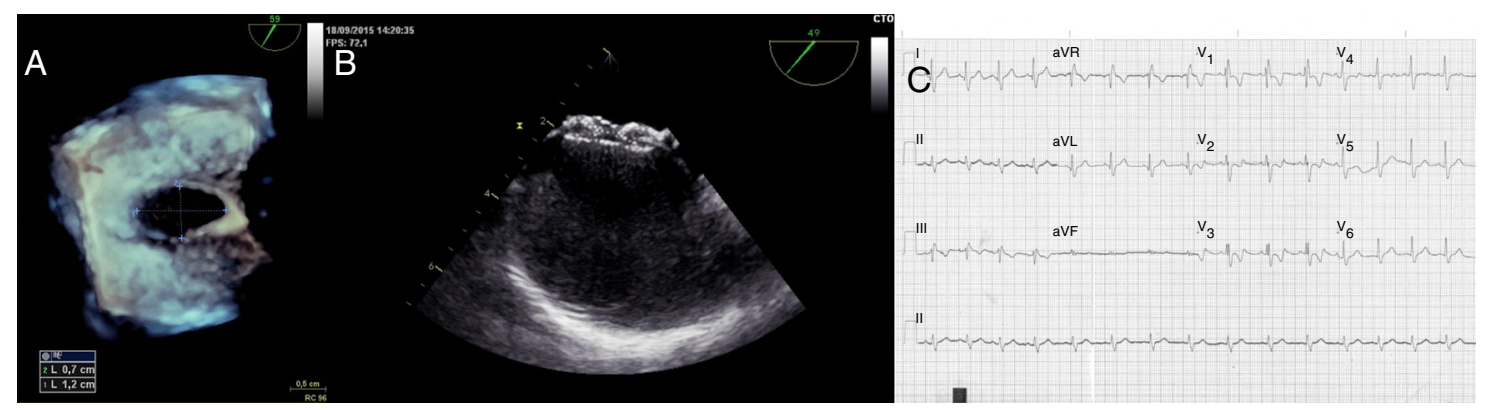

Figura 2 A) Dimensiones del defecto del septum por ecocardiograma tridimensional. B) Dispositivo ocluyendo el defecto. C) Electrocardiograma con ritmo sinusal y conducción AV conservada al mes de seguimiento después del cierre del defecto.

después de 2 meses de colocados los dispositivos, lo que sugiere que dichos dispositivos influirían de alguna forma en la conducción intraauricular ${ }^{3}$. Hill et al. sugieren que un mecanismo posible podría ser la presión continua que ejerce el dispositivo o la fricción del disco derecho sobre el nódulo $\mathrm{AV}$, induciendo edema de pared y posterior bloqueo del nódulo. Por otra parte, la mayoría de los bloqueos AV aparecen durante el procedimiento o en los primeros días de ocluido, y en general son transitorios, recuperándose la conducción AV espontáneamente o con uso de corticoides, concordando con la teoría fisiopatológica del edema peri nódulo $\mathrm{AV}^{2}$. Suda et al., en una experiencia de 162 pacientes, reportan 2 casos de bloqueo $\mathrm{AV}$ completo reversibles, ocurridos intraprocedimento, los cuales recuperaron la conducción $\mathrm{AV}$ normal, en un caso a las $24 \mathrm{~h}$ y en el otro a las
2 semanas $^{4}$, con menor frecuencia, la conducción AV no se recupera y es necesario el uso de marcapasos definitivo ${ }^{5}$.

El sobredimensionamiento del dispositivo y la presencia de bordes deficientes, principalmente el inferoposterior, han sido otros factores de riesgo posiblemente asociados a trastornos de conducción ${ }^{6}$. La edad y el peso del paciente están en íntima relación con estos factores de riesgo, sobre todo con la posibilidad de sobredimensionamiento, aunque en nuestro caso la relación entre el tamaño del defecto y el peso era menor a 1,5 veces $^{7}$.

Con respecto al tratamiento, se propone el uso de antiinflamatorios y corticoides, y son mucho menos frecuentes los casos de requerimiento de cirugía para extracción de dispositivo o la necesidad de implante de marcapasos definitivo ${ }^{6}$, cuando la conducción AV no se recupera. 
Consideramos que el paciente que se presenta es importante por 2 motivos, el primero el ser el primer bloqueo AV transitorio con dispositivo Nit Occlud ${ }^{\circledR}$, y segundo, mostrar una alternativa al tratamiento, que es intentar el cierre diferido con un dispositivo de menor tamaño ${ }^{4,6}$. Esta estrategia requiere seguimiento estricto a largo plazo, ya que se han reportado casos de bloqueo AV completo hasta 4 años después del implante del dispositivo ${ }^{8}$.

\section{Financiación}

No se recibió patrocinio de ningún tipo para llevar a cabo este artículo.

\section{Bibliografía}

1. Chessa M, Carminati M, Butera G, et al. Early and late complications associated with transcatheter occlusion of secundum atrial septal defect. J Am Coll Cardiol. 2002;39:1061-5.

2. Hill SL, Berul CI, Patel HT, et al. Early ECG abnormalities associated with transcatheter closure of atrial septal defects using the Amplatzer septal occluder. J Interv Card Electrophysiol. 2000;4:469-74.

3. Johnson JN, Marquardt ML, Ackerman MJ, et al. Electrocardiographic changes and arrhythmias following percutaneous atrial septal defect and patent foramen ovale device closure. Catheter Cardiovasc Interv. 2011;78:254-61.

4. Suda K, Raboisson MJ, Piette E, et al. Reversible atrioventricular block associated with closure of atrial septal defects using the Amplatzer device. J Am Coll Cardiol. 2004;43:1677-82.

5. Du ZD, Hijazi ZM, Kleinman CS, et al. Comparison between transcatheter and surgical closure of secundum atrial septal defect in children and adults. J Am Coll Cardiol. 2002;39:1836-44.
6. Al-Anani SJ, Weber H, Hijazi ZM. Atrioventricular block after transcatheter ASD closure using the Amplatzer septal occluder: Risk factors and recommendations. Catheter Cardiovasc Interv. 2010;75:767-72.

7. Moore J, Hedge S, El-Said H, et al. Transcatheter device closure of atrial septal defects. J Am Coll Cardiol Cardiovasc Interv. 2013;6:433-42.

8. Nehgme RA, Huddleston AR, Cheatham JP. Progression to late complete atrioventricular block following Amplatzer device closure of atrial septal defect in a child. Pediatr Cardiol. 2009;30:367-70.

Alejandro E. Contreras ${ }^{a, *}$, Alejandro Peirone ${ }^{b}$, Adolfo Ferrero Guadagnoli ${ }^{\mathrm{b}}$ y Jonathan Miara Lopez ${ }^{\mathrm{b}}$

a Servicio de Cardiología, Hospital Privado Universitario de Córdoba, Instituto de Ciencias Biomédicas de Córdoba, Córdoba, Argentina

${ }^{\mathrm{b}}$ Servicio de Hemodinamia, Hospital Privado Universitario de Córdoba, Instituto de Ciencias Biomédicas de Córdoba, Córdoba, Argentina

* Autor para correspondencia. Hospital Privado Universitario, Naciones Unidas 346, CP 5016, Córdoba, Argentina; Teléfono: +54 3514688220; Fax: +54 3514688818.

Correo electrónico: aletreras@hotmail.com

(A.E. Contreras).

http://dx.doi.org/10.1016/j.acmx.2016.05.005 1405-9940/

(c) 2016 Instituto Nacional de Cardiología Ignacio Chávez. Publicado por Masson Doyma México S.A. Este es un artículo Open Access bajo la licencia CC BY-NC-ND (http:/ / creativecommons.org/ licenses/by-nc-nd/4.0/).

\section{Hipertensión pulmonar secundaria a enfermedad de Rendu-Osler-Weber. Reporte de caso}

\section{Pulmonary hypertension in Rendu-Osler-Weber disease. A case report}

La telangiectasia hemorrágica hereditaria $(\mathrm{THH})$ o enfermedad de Rendu-Osler-Weber, es una condición poco frecuente caracterizada por múltiples telangiectasias y malformaciones arteriovenosas que pueden llevar a episodios repetitivos de epistaxis y otros tipos de sangrado. Las manifestaciones clínicas en la THH son causadas por el desarrollo de la vasculatura anormal (incluyendo telangiectasias, malformaciones arteriovenosas y aneurismas). Se han identificado mutaciones de herencia autosómico dominante en al menos 3 genes: endoglina (ENG), ACVRL1 (HHT2) y SMAD4, y las mutaciones en los primeros 2 explican el $85 \%$ de los casos $^{1}$.

La presentación clínica consiste en epistaxis de repetición, así como en telangiectasias mucocutáneas y malformaciones arteriovenosas viscerales. El diagnóstico se basa en los criterios de Curaçao $^{2}$ (tabla 1). Si bien las alteraciones mucocutáneas y las malformaciones arteriovenosas son las manifestaciones distintivas del síndrome de Rendu-Osler-Weber, también se ha reportado la asociación de hipertensión pulmonar (HP) como una complicación importante. Reportamos el caso de una paciente con síndrome de Rendu-Osler-Weber e HP.

Mujer de 42 años que acudió a consulta a nuestro instituto con historia de 2 años de disnea de grandes esfuerzos y fatiga. Entre sus antecedentes patológicos se incluía un embarazo complicado por preeclampsia a los 40 años de edad y sangrado uterino anómalo de repetición. Negaba consumo de medicamentos o drogas. Al interrogatorio dirigido destacaba la presencia de epistaxis recurrente desde los 23 años de edad. El examen físico demostró la presencia de numerosas telangiectasias en cavidad oral y en miembros pélvicos (fig. 1). El examen del precordio con $2 \mathrm{P}$ reforzado y soplo holosistólico grado ॥/ıv en foco tricúspide. El electrocardiograma en ritmo sinusal, con ondas $\mathrm{P}$ pulmonares y crecimiento del ventrículo derecho. Un ecocardiograma transtorácico mostró crecimiento de cavidades derechas, presión sistólica arterial pulmonar de $52 \mathrm{mmHg}$ e insuficiencia tricuspídea moderada a severa. Se realizó una tomografía toracoabdominal que demostró numerosas malformaciones arteriovenosas a nivel de cuello, hígado y bazo (fig. 2). Se realizó el diagnóstico de síndrome de Rendu-Osler-Weber. Ante la sospecha de HP, se realizó un cateterismo derecho 